\title{
Is there a relationship between blood lipids and lumbar disc herniation in young Turkish adults?
}

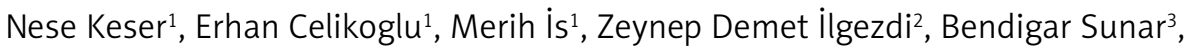
Yusuf Sinan Aydin ${ }^{2}$, Ahmet Ugur Kevenk', Bora Gurer ${ }^{1}$, Ali Fatih Ramazanoglü ${ }^{5}$, Nurgul Keser ${ }^{6}$

1Department of Neurosurgery, TR University of Health Sciences, Istanbul Fatih Sultan
Mehmet Education and Research Hospital, Istanbul, Turkey
2Department of Cardiology, TR University of Health Sciences, Istanbul Fatih Sultan
Mehmet Education and Research Hospital, Istanbul, Turkey
${ }^{3}$ Department of Biochemistry, TR University of Health Sciences, Istanbul Fatih Sultan
Mehmet Education and Research Hospital, Istanbul, Turkey
${ }^{4}$ Department of Health Management, Istanbul Bilgi University Faculty of Health
Sciences, Istanbul, Turkey
${ }^{5}$ Department of Neurosurgery, TR University of Health Sciences, Umraniye Education
and Research Hospital, Istanbul, Turkey
${ }^{6}$ Department of Cardiology, University of Sakarya Faculty of Medicine, Sakarya, Turkey

Submitted: 29 May 2017

Accepted: 12 June 2017

Arch Med Sci Atheroscler Dis 2017; 2: e24-e28

DOI: https://doi.org/10.5114/amsad.2017.68651

Copyright @ 2017 Termedia \& Banach

\section{Abstract}

Introduction: Atherosclerosis might diminish the nutrient supply to intervertebral discs (IVD), leading to disc herniation. Therefore, there is interest in determining the possible association between the blood lipid profile and lumbar disc herniation (LDH). We aimed to evaluate the association between blood lipids and LDH in a homogeneous group of patients, controlling for age- and sex-specific effects.

Material and methods: This is a case-control study which consisted of 100 individuals (mean age: $41.25 \pm 9.09 ; 50$ men and 50 women), classified into two groups, as follows. Group I (G-I) consisted of 50 patients who underwent surgery for symptomatic LDH, while group II (G-II) consisted of 50 patients with nonspecific complaints of a headache, but with no previous history of back and/or leg pain, recruited among patients admitted to the outpatient clinic at the time of the study, and whose age and sex were matched to the study group. Total cholesterol (TC), triglyceride (TG), low-density lipoprotein cholesterol (LDL-C), high-density lipoprotein cholesterol (HDL-C), fasting blood glucose, and hemoglobin $\mathrm{A}_{1 \mathrm{c}}$ levels were measured. The TC/HDL-C ratio was calculated. Blood pressure, waist circumference, body mass index, and the history of smoking were included in the analysis.

Results: The mean values of the TC, TG, LDL-C, HDL-C levels and TC/HDL-C ratio were $198.38,132.76,131.9,40.38 \mathrm{mg} / \mathrm{dl}$ and 5.09 , respectively. No statistically significant relationship between the blood lipid profile and LDH was identified in this population.

Conclusions: Blood lipid levels in this young adult Turkish population did not predict $L D H$, and may not be a leading cause of IVD ischemia and IVD degeneration.

Key words: atherosclerosis, lipids, gender, intervertebral disc degeneration, lumbosacral region.

\author{
Corresponding author: \\ Nese Keser \\ Departement of \\ Neurosurgery \\ TR University \\ of Health Sciences \\ Istanbul Fatih Sultan \\ Mehmet Education \\ and Research Hospital \\ 34752 Istanbul, Turkey \\ Phone: +90 5323326407 \\ Fax: +90 2165750406 \\ E-mail: nskeser@gmail.com
}




\section{Introduction}

Back problems are the leading cause of work-related disability, with the majority of adults reporting at least one episode of back pain during their lifetime [1]. Disc herniation, resulting from intervertebral disc degeneration (IVDD) and mechanical loading, is a primary cause of back pain, affecting both men and women. The IVDD, which is an expression of the state of the disc, has a complex and multifactorial etiology. Various factors have been identified as contributing to IVDD, including genetics [2]; toxic factors, such as smoking [3]; nutritional status [4]; mechanical factors, such as repetitive compressive loading [5]; autoimmune responses [6]; aging [7]; and metabolic factors, such as diabetes mellitus (DM) [8]. While most of these factors act individually or collectively, what remains unexplained is how they affect the intervertebral disc (IVD). Atherosclerotic plaques in the abdominal aorta and congenital hypoplasia of lumbar arteries diminish blood flow, and consequently nutrient flow, to IVDs, leading to IVDD and disc herniation, secondary to ischemic effects [9]. Whether or not cardiovascular risk factors (CVRFs), used as surrogate markers for atherosclerosis, might also be a marker of diminished nutrient supply to IVDs is an interesting research area. Blood lipids constitute a major component of classical CVRFs [10]. However, there have been conflicting reports regarding the association between blood lipids and IVDD [11, 12]. Therefore, the blood lipid parameters, and their threshold, that might predict disc ischemia and subsequent IVDD remain to be identified.

Additionally, clear sex-specific differences in coronary artery disease (CAD) have been reported, with estrogen providing a protective effect to women with regard to the age of onset and symptoms of atherosclerosis, as well as influencing the effect of CVRFs and mechanisms of microvascular dysfunction [13]. Based on this information, we propose that conflicting views regarding the association between CVRFs and IVDD might be related to the enrollment of patients over a wide age range, without careful control for sex-specific effects, and not including all potential blood lipid parameters.

Thus, our aim in this study was to evaluate all major blood lipid parameters in a homogeneous group of patients under the age of 60 years, with an equal number of men and women with symptomatic lumbar disc herniation (LDH) included.

\section{Material and methods}

\section{Study design and population}

After receiving ethical approval and informed consent from each patient, 100 patients were en- rolled in the study and divided into two groups. Group I (G-I) included 50 patients (25 men, 25 women; mean age, $41.12 \pm 1.16$ ) with symptomatic LDH, who had undergone surgical treatment at our clinic (University of Health Sciences, Istanbul Fatih Sultan Mehmet Education and Research Hospital Neurosurgery Department), between December 2013 and June 2014. Group II (G-II) included 50 patients (25 men, 25 women; mean age: $41.38 \pm 1.41$ ) with complaints of nonspecific headache but with no history of back and/ or leg pain, who were recruited among patients admitted to our outpatient clinic at the time of the study, and whose age and sex were matched to the study group.

The inclusion criteria for G-I were as follows: unilateral radicular leg pain; positive signs of nerve root irritation ( $L 3, L 4, L 5$ and/or S1) on the straight leg raising or femoral stretch test; neurological signs associated with radiculopathy, presenting as muscle weakness or decrease/absence of corresponding reflexes; confirmation of IVDD and disc herniation by magnetic resonance imaging (MRI); non-response to medical intervention; progressive motor deficit, with abnormal electromyographic (EMG); and absence of accompanying headache of any type. The inclusion criteria for G-II were as follows: no history of lower back and leg pain; no prior clinical diagnosis of disc herniation; and a diagnosis of a nonspecific headache. Patients with herniation of multiple intervertebral discs, spondylolisthesis, central or foraminal stenosis, spondyloarthritis, cerebrovascular disease, hypertension, diabetes mellitus (DM), coronary heart disease, a body mass index (BMI) $>30 \mathrm{~kg} / \mathrm{m}^{2}$, as well as women in menopause and those who used any drugs, were excluded from the study. Fasting total blood cholesterol (TC), triglyceride (TG), low-density lipoprotein cholesterol (LDL-C), high-density lipoprotein cholesterol (HDL-C), blood glucose and hemoglobin $A_{1 c}\left(\mathrm{HbA}_{1 c}\right)$ levels were obtained. All blood samples were collected in our laboratory using a vacutainer system (Becton-Dickinson, NJ, USA). Samples from all participants were obtained in the morning, after a 10-h overnight fast. For biochemical tests (Glucose, TC, TG, HDL-C, and LDL-C), $5 \mathrm{~mm}$ blood samples were centrifuged for $10 \mathrm{~min}$ at $3000 \mathrm{rpm}$. Sera were then extracted and analyzed using the enzymatic methods of Abbott Laboratories (Architect C16000 model auto-analyser; Abbott Laboratories, Abbott Park, IL, USA). LDL-C (mg/dl) was calculated using the Friedewald formula for patients with TG levels < $400 \mathrm{mg} / \mathrm{dl}$ : LDL-C = TC - HDL-C - TG/5 [14]. Normal values were defined according to the ATPIII guidelines [15], as follows: TC $<200 \mathrm{mg} / \mathrm{dl}$; LDL-C < $130 \mathrm{mg} / \mathrm{dl}$; HDL-C > $40 \mathrm{mg} / \mathrm{dl}$; and TG $<150 \mathrm{mg} / \mathrm{dl}$. The TC/HDL ratio was calculated for each patient. For the analysis of $\mathrm{HbA}_{1 \mathrm{c}}$, blood 
samples were collected in EDTA tubes and evaluated using the HPLC method (Premier Hb9210 model instrument, Trinity Biotech, Bray, Co Wicklow, Ireland). Fasting blood glucose $>126 \mathrm{mg} / \mathrm{dl}$ and $\mathrm{HbA}_{1 \mathrm{c}}>6.5 \%$ were considered to be abnormal [16]. Waist circumference (WC), weight, and height were measured by the same physician, and the BMI calculated. Waist circumference was measured with the patient in a standing position, at the level of the umbilicus just above the iliac crests. The BMI $\left(\mathrm{kg} / \mathrm{m}^{2}\right)$ was classified according to the World Health Organization criteria, as follows: 25-30, overweight; 30-40, obese; and > 40, morbidly obese [17]. Abdominal obesity was identified according to the National Heart, Lung and Blood Institute criteria [18]: $W C \geq 102 \mathrm{~cm}$ in men, $W C$ $\geq 88 \mathrm{~cm}$ in women and a BMI $>30 \mathrm{~kg} / \mathrm{m}^{2}$. Systolic blood pressure (SBP) and diastolic blood pressure (DBP) were measured from the right arm, with the patient in a sitting position, through indirect auscultation, using a calibrated manometer with a $14 \times 40 \mathrm{~cm}$ cuff. Based on the ASH/ISH 2014 criteria of a pressure of $140 / 90 \mathrm{~mm} \mathrm{Hg}$ as a threshold for HT diagnosis for patients < 60 years old, we classified participants with a blood pressure $>140 / 90 \mathrm{~mm} \mathrm{Hg}$ as being hypertensive [19].

A careful history was taken for current/past smoking. Smoking was simply evaluated as a binary response, as follows: ' 0 ', never smokes, or stopped smoking on average five years ago; ' 1 ', current and regular smoker.

\section{Statistical analysis}

Statistical analysis was performed using the IBM SPSS Statistics program (version 22; IBM SPSS, Turkey). The normal distribution of the data- set was evaluated using the Shapiro-Wilk test. Descriptive statistics (mean, standard deviation and frequency) were calculated for each parameter. Between-group differences were evaluated using Student's t-test for normally distributed data and the Mann-Whitney $U$ test for data with a non-normal distribution. Between-group differences in qualitative data were evaluated using the $\chi^{2}$ test or Fisher's exact test, as appropriate. A $p$-value $<0.05$ was considered statistically significant.

\section{Results}

Baseline characteristics of participants are shown in Table I. The LDH group consisted of 50 cases ( 25 males and 25 females), with a mean age of 41.12 years and BMI of $27.95 \mathrm{~kg} / \mathrm{m}^{2}$. The control group consisted of 50 patients $(25$ males and 25 females), with a mean age of 41.38 years and BMI of $28 \mathrm{~kg} / \mathrm{m}^{2}$. We purposefully did not include menopausal women in either the study or control groups. The study and control groups had a comparable distribution of age, BMI, WC, BP levels and smoking rate $(p>0.05)$.

Serum concentrations of TC, TG, LDL-C, HDL-C, blood glucose and $\mathrm{HbA}_{1 c}$ levels for both groups are summarized in Table II. All levels were within normal limits, with no significant between-group differences noted. Therefore, no association between serum levels of TC, TG, LDL-C, HDL-C levels, TC/ HDL-C ratio and LDH was identified in our study population.

\section{Discussion}

The IVD is the largest avascular tissue in the body, receiving its nutrients via the capillary net-

Table I. Evaluating the operating parameters according to the study (G-I) and control (G-II) groups in all cases

\begin{tabular}{|lcccc|}
\hline Parameter & $\begin{array}{c}\text { G-I (disc herniation) } \\
(n=50)\end{array}$ & $\begin{array}{c}\text { G-II (control) } \\
(n=50)\end{array}$ & $\begin{array}{c}95 \% \text { confidence interval } \\
\text { of the difference }\end{array}$ & $P$-value \\
\hline Age & $41.12 \pm 1.16$ & $41.38 \pm 1.41$ & $-3.88-3.36$ & $0.887^{1}$ \\
\hline BMI $\left[\mathrm{kg} / \mathrm{m}^{2}\right]$ & $27.95 \pm 0.66$ & $28.79 \pm 0.56$ & $-2.55-0.87$ & $0.332^{1}$ \\
\hline WC $[\mathrm{cm}]$ & $95.74 \pm 1.77$ & $96.76 \pm 1.44$ & $-5.55-3.51$ & $0.656^{1}$ \\
\hline SBP & $124.6 \pm 13.06$ & $122.78 \pm 15.5$ & $-3.87-7.51$ & $0.527^{1}$ \\
\hline DBP & $75.64 \pm 9.82$ & $74.96 \pm 10.31$ & $-3.32-4.68$ & $0.736^{1}$ \\
\hline \begin{tabular}{l} 
Gender: \\
\hline Male
\end{tabular} & $25(25 \%)$ & $25(25 \%)$ & & $1.000^{2}$ \\
\hline Female & $25(25 \%)$ & $25(25 \%)$ & & \\
\hline Smoking: & $13(26 \%)$ & $18(36 \%)$ & & $0.387^{2}$ \\
\hline Yes & $37(74 \%)$ & $32(64 \%)$ & & \\
\hline No & & & \\
\hline
\end{tabular}

${ }^{1}$ Student's t-test, ${ }^{2}$ Continuity (Yates) correction, BMI - body mass index, WC - waist circumference, SBP - systolic blood pressure, DBP diastolic blood pressure. 
Table II. Evaluating biochemical parameters according to the study (G-I) and control (G-II) groups in all cases

\begin{tabular}{|lcccc|}
\hline Parameter & $\begin{array}{c}\text { G-I (disc herniation) } \\
(n=50)\end{array}$ & $\begin{array}{c}\text { G-II (control) } \\
(n=50)\end{array}$ & $\begin{array}{c}\text { 95\% confidence interval } \\
\text { of the difference }\end{array}$ & $P$-value \\
\cline { 2 - 3 } & Mean \pm SD & Mean \pm SD & & \\
\hline FBG & $96.7 \pm 14.42$ & $94.44 \pm 7$ & $-2.26-6.78$ & $0.322^{1}$ \\
\hline HbA ${ }_{1 C}$ & $5.43 \pm 0.47$ & $5.4 \pm 0.31$ & $-0.12-0.19$ & $0.651^{1}$ \\
\hline TC & $198.38 \pm 41.27$ & $199.1 \pm 41.29$ & $-17.10-15.66$ & $0.931^{1}$ \\
\hline TG (median) & $132.76 \pm 65.83(113)$ & $134.58 \pm 69.89(112.5)$ & $-28.77-25.13$ & $0.863^{2}$ \\
\hline LDL-C & $131.9 \pm 35.31$ & $129.88 \pm 32.76$ & $-11.49-15.54$ & $0.767^{1}$ \\
\hline HDL-C & $40.38 \pm 9.5$ & $43.28 \pm 9.17$ & $-6.60-0.80$ & $0.124^{1}$ \\
\hline TC/HDL-C & $5.09 \pm 1.29$ & $4.28 \pm 1.57$ & $-2.97-0.84$ & $0.344^{1}$ \\
\hline
\end{tabular}

${ }^{1}$ Student $t$-test, ${ }^{2}$ Mann-Whitney U test. FBG - fasting blood glucose, TC-total cholesterol, TG - triglyceride, LDL-C - low-density lipoprotein cholesterol, HDL-C - high-density lipoprotein cholesterol.

work and relying on diffusion through the vertebral endplate [20]. Since ischemia is capable of causing both pain and degeneration of the involved structures, an atherothrombotic disease of the supplying arteries of the lumbar spine has received increasing attention as one of the possible underlying factors for both lumbar back pain and IVDD. Previous studies have provided evidence of a correlation between decreased diffusion of the lumbar disc and diminished blood flow in the corresponding lumbar arteries [21, 22].

In our study, we evaluated TC, TG, LDL-C, and HDL-C levels, and TC/HDL-C ratio, among patients with symptomatic LDH, controlling for effects of age and sex, and compared these parameters to a control group of patients of a similar age with nonspecific complaints. No between-group differences were identified. As there is little current information available regarding the association between blood lipid parameters, either as a group or independent parameters, and IVDD, evaluation of our results relative to previous research was limited. Our results contrast with the findings of Longo et al. [12], who reported a moderate increase in levels of TG (162 mg/dl) and TC (215 mg/ dl) among patients, 59 years old on average, with $\mathrm{LDH}$. This difference between studies is likely a result of the inclusion of older individuals by Longo et al. and differences in the distribution of males and females. Also, using only TC levels to predict atherosclerotic risk can be misleading, particularly for women who often have high HDL-C levels. For an adequate atherosclerotic risk analysis, it is recommended that at least both $\mathrm{HDL}-\mathrm{C}$ and LDL-C be analyzed [14], in combination with TC levels, as we have done in our study. As an example, although a previous study reported an association between serum TC and sciatic pain among men [21], in women, high TC levels were found to be associated with LDH [23], emphasizing the impor- tance of controlling for sex effects. The study by Zhang et al. [24] included patients with a similar mean age as our study, but with at $1: 1.5$ distribution of women to men. As we previously stated, the impact of menopause on the blood lipid profile and the interaction between atherosclerosis and symptomatic LDH need to be considered. In Longo et al.'s study [12], the TG level of 162 $\mathrm{mg} / \mathrm{dl}$ was found to be associated with $\mathrm{LDH}$. This is in contrast to our study, where there was no difference in TG levels between the groups, with a mean value of $132 \mathrm{mg} / \mathrm{dl}$. However, it should be remembered that TG appears to influence CAD risk in older women uniquely, especially at levels $>400 \mathrm{mg} / \mathrm{dl}$ [25].

Within the CVRF profile, obesity and excess body weight are also important factors to consider and have been reported to be associated with radiating lumbar pain or $\mathrm{LDH}$, due to both mechanical loading and via atherosclerosis [5]. In our study, the mean BMI was below $30 \mathrm{~kg} / \mathrm{m}^{2}$ for both men and women, with no difference between groups. However, the WC was comparable between men and women, which was indicative of obesity among women in our study group according to IDF [26] and AHA/ NHLBI [18] criteria. The interaction between WC and LDH has not been previously evaluated.

Smoking, which is also an important CVRF for both sexes, has previously been associated with increased incidence of LDH and IVDD [3]. In our study, there was also no difference between the groups regarding smoking. However, because of the low prevalence of smoking in our study group, the association between smoking and LDH could not be reliably evaluated.

The strengths of our study include standardized analysis of fasting blood samples and preoperative imaging of $\mathrm{LDH}$. Moreover, we evaluated not only the TG and TC levels but also HDL-C and LDL-C levels and the TC/HDL ratio. Moreover, we 
controlled for known effects of hypertension and DM by excluding these individuals, and used a homogeneous study sample, with equal distribution of men under the age of 60 years and women who are not in menopause, thereby also controlling for effects of aging and menopause on lipid parameters. However, our sample size was small, which prevented subgroup analyses. Further studies should consider the effect of each CVRF separately, with attention paid to clustering effects between parameters.

In conclusion, we did not find any relationship between blood lipid profiles and LDH in our study population. As the types and levels of lipids associated with LDH may be different in different population groups, there is a need for further studies in this area. Identification of a reliable blood lipid profile associated with IVD would help to elucidate the pathogenesis of IVDD and contribute to the prevention and treatment of $\mathrm{LDH}$.

\section{Conflict of interest}

The authors declare no conflict of interest.

\section{References}

1. Kauppila LI. Atherosclerosis and disc degeneration/lowback pain - a systematic review. Eur J Vasc Endovasc Surg 2009; 37: 661-70.

2. Sambrook PM, MacGregor AJ, Spector TD. Genetic influences on cervical and lumbar disc degeneration: a magnetic resonance imaging study in twins. Arthritis Rheum 1999; 42: 366-72.

3. Akmal M, Kesani A, Anand B, Singh A, Wiseman M, Goodship A. Effect of nicotine on spinal disc cells: a cellular mechanism for disc degeneration. Spine 2004; 29: 568-75.

4. Benneker LM, Heini PF, Alini M, Anderson SE, Ito K. 2004 Young Investigator Award Winner: vertebral endplate marrow contact channel occlusions and intervertebral disc degeneration. Spine 2005; 30: 167-73.

5. Pye SR, Reid DM, Adams JE, Silman AJ, O'Neill TW. Influence of weight, body mass index and lifestyle factors on radiographic features of lumbar disc degeneration. Ann Rheum Dis 2007; 66: 426-27.

6. Wang J, Tang T, Yang H, et al. The expression of Fas ligand on normal and stabbed-disc cells in a rabbit model of intervertebral disc degeneration: a possible pathogenesis. J Neurosurg Spine 2007; 6: 425-30.

7. Roberts S, Evans EH, Kletsas D, Jaffray DC, Eisenstein SM. Senescence in human intervertebral discs. Eur Spine J 2006; 15 (Suppl 3): S312-6.

8. Robinson D, Mirovsky Y, Halperin N, Evron Z, Nevo Z. Changes in proteoglycans of intervertebral disc in diabetic patients. A possible cause of increased back pain. Spine 1998; 23: 849-55.

9. Kauppila LI, Tallroth K. Postmortem angiographic findings for arteries supplying 1 the lumbar spine: their relationship to low-back symptoms. J Spinal Disord 1993; 6: 124-29.

10. Kumar A, Cannon CP. Acute coronary syndromes: diagnosis and management, part I. Mayo Clin Proc 2009; 84: 917-38.
11. Leino-Arjas P, Kauppila L, Kaila-Kangas L, Shiri R, Heistaro S, Heliövaara M. Serum lipids in relation to sciatica among Finns. Atherosclerosis 2008; 197: 43-9.

12. Longo UG, Denaro L, Spiezia F, Forriol F, Maffulli N, Denaro V. Symptomatic disc herniation and serum lipid levels. Eur Spine J 2011; 20: 1658-62.

13. Kulkarni R, Kulkarni A. Cardiovascular disease in women: myths and facts about a silent epidemic. In: Heart Disease in Women. Nanda NC, Keser N (eds). Jaypee Publishers, New Delhi 2015; 3-9.

14. Reiner Z, Catapano AL, Backer GD, et al. ESC/EAS Guidelines for the management of dyslipidaemias. The Task Force for the management of dyslipidaemias of the European Society of Cardiology (ESC) and the European Atherosclerosis Society (EAS). Eur Heart J 2011; 32: 1769-818.

15. Third report of the National Cholesterol Education Program Expert Panel on Detection, Evaluation, and Treatment of High Blood Cholesterol in Adults (Adult Treatment Panel III). Circulation 2002; 106: 3143-421.

16. American Diabetes Association. Diagnosis and classification of diabetes mellitus. Diabetes Care 2010; 33 (Suppl 1): S62-9.

17. WHO website:http://www.who.int/bmi

18. Grundy SM, Cleeman JI, Daniels SR, et al. Diagnosis and management of the metabolic syndrome: an American Heart Association/National Heart, Lung, and Blood Institute Scientific Statement. Circulation 2005; 112: 2735-52.

19. Krakoff LR, Gillespie RL, Ferdinand KC, et al. $2014 \mathrm{Hy}$ pertension Recommendations From the Eighth Joint National Committee Panel Members Raise Concerns for Elderly Black and Female Populations. J Am Coll Cardiol 2014; 64: 394-402.

20. Stairmand JW, Holm S, Urban JP. Factors influencing oxygen concentration gradients in the intervertebral disc. A theoretical analysis. Spine 1991; 16: 444-9.

21. Kauppila LI, Mikkonen R, Mankinen P, Pelto-Vasenius K, Mäenpää I. MR aortography and serum cholesterol levels in patients with long-term nonspecific lower back pain. Spine 2004; 29: 2147-52.

22. Tokuda O, Okada M, Fujita T, Matsunaga N. Correlation between diffusion in lumbar intervertebral disks and lumbar artery status: evaluation with fresh blood imaging technique. J Magn Reson Imaging 2007; 25: 185-91.

23. Jhawar BS, Fuchs CS, Colditz GA, Stampfer MJ. Cardiovascular risk factors for physician-diagnosed lumbar disc herniation. Spine J 2006; 6: 684-91.

24. Zhang Y, Zhao Y, Wang M, Stampfer MJ. Serum lipid levels are positively correlated with lumbar disc herniation - a retrospective study of 790 Chinese patients. Lipids Health Dis 2016; 15: 80.

25. Tokgozoglu L, Canpolat U. Dyslipidemia in women. In: Heart Disease in Women. Nanda NC, Keser N (eds). Jaypee Publishers, New Delhi 2015: 30-5.

26. International Diabetes Federation. The IDF consensus worldwide definition of the metabolic syndrome. Brussels 2006 